\title{
Operatividad Interna de Movilidad Internacional en una Universidad Pública Mexicana
}

\section{Internal Operation of International Mobility at a Mexican Public University}

\section{Operatividad Interna de Movilidad Internacional}

\author{
Alejandro García Vidal ${ }^{(\mathbf{1})}$ \\ Gladys Hernández Romero ${ }^{(2)}$ \\ (1) Universidad Juárez Autónoma de Tabasco. México. email: alex_garvi95@live.com.mx \\ (2) Universidad Juárez Autónoma de Tabasco.México. email: $\underline{\text { doctoragladys@ @otmail.com }}$ \\ Contacto: \\ alex_garvi95@live.com.mx
}

Recibido: 21-9-2020

Aprobado: 20-11-2020

\section{Resumen}

El intercambio estudiantil se ha convertido en una estrategia de formación para las universidades debido a la globalización, permitiendo que los alumnos amplíen sus conocimientos. El presente artículo tiene como objetivo determinar la operatividad interna del Programa de Movilidad Estudiantil en la Universidad Juárez Autónoma de Tabasco. El método utilizado fue empírico y se basó en una muestra intencional de 103 cuestionarios aplicados a estudiantes cuyo avance curricular se encuentra entre el $50 \%$ y el $96 \%$. Los principales resultados destacan que el $100 \%$ de alumnos tiene conocimiento sobre la existencia del programa y la mayoría prefiere su estancia en países donde no se les dificulte el idioma, por lo que el $31 \%$ de ellos optó por viajar a países del continente americano. Uno de los beneficios que se obtiene de la movilidad, según señalan los universitarios, es el desarrollo académico y personal. El programa de Movilidad impulsa la formación académica, abriendo paso a la competitividad que exige el campo laboral y a la vez permite que los estudiantes aprendan sobre la cultura de otros países.

Palabras clave: Movilidad estudiantil, estrategias educativas, aprendizaje, cultura, competencia profesional

\section{Abstract}

Student exchange has become a training strategy for universities due to globalization, allowing students to expand their knowledge. The objective of this article is to determine the internal operation of the Student Mobility Program at the Universidad Juárez Autónoma de Tabasco. The method used was empirical and was based on an intentional sample of 103 questionnaires applied to students whose curricular progress was between $50 \%$ and $96 \%$. The main results highlight that $100 \%$ of students are aware of the existence of the program and most prefer to stay in countries where the language is not difficult for them, so $31 \%$ of them chose to travel to countries in the American continent. One of the benefits obtained from mobility, according to university students, is academic and personal development. The Mobility program promotes academic training, opening the way to the competitiveness required by the labor field and at the same time allows students to learn about the culture of other countries.

Keywords: Student mobility, educational strategies, learning, culture, professional competence.

\section{Introducción}

Sin lugar a dudas los programas académicos que implican desplazamiento o movilidad de personas, suponen también un dinamismo humano, las más de las veces imparable. García (2013) menciona que la movilidad académica "... hace referencia al desplazamiento de investigadores, docentes y alumnos entre Instituciones Educativas nacionales y 
extranjeras con el objetivo de participar en programas formativos y proyectos de investigación particulares.". Dentro de la Universidad Juárez Autónoma de Tabasco este programa ha dado un gran paso en los últimos años, ofreciendo oportunidades de crecimiento estudiantil a los jóvenes que desean una fructífera y exitosa preparación profesional.

En el Informe presentado por la UNESCO sobre ciencia (2015), se destaca el impacto de la inversión para la investigación y desarrollo, cómo influyen en el desarrollo de las naciones y cómo éstas han promovido la investigación durante la formación universitaria, dando origen a la mejora curricular, ofreciendo incentivos para la generación de más y mejores profesionistas. Esto ha dado origen a una economía mundial del conocimiento, como resultado a la apertura de fronteras a estudiantes, con el fin de la utilización y combinación innovadora de conocimientos de otras procedencias.

Aunado a lo anterior y de acuerdo con Torres, Medina, y Alvarado (2019) en México se han instaurado políticas competentes a la movilidad académica y el desarrollo de la ciencia y tecnología. Algunas son prácticas con el objetivo de fomentar el intercambio de experiencias y diversificación en la formación del alumnado de las Instituciones de Educación Superior (IES). Otras incentivan la formación de profesionales que desarrollen nuevos conocimientos y en consecuencia se genere ciencia y tecnología que impulsen el desarrollo del país.

En la actualidad, gracias a la globalización y a los enormes cambios culturales, sociales y económicos, los universitarios tienen mejores oportunidades académicas donde se les ofrece estudiar en otro estado u otro país para enriquecer sus conocimientos y llenarse de experiencias, aprendizajes y enseñanzas que le serán de ayuda en su formación profesional.

González (2011) comparte que los desplazamientos son originados por el deseo de mejora en el desarrollo profesional. Al llevarse a cabo la movilidad tanto nacional como internacional se crea un intercambio de culturas, pensamientos y sobre todo conocimientos entre los estudiantes que los conducen a un crecimiento personal, académico y profesional.

Belvis, Pineda y Moreno (2007) afirman que el participar en una actividad académica como ésta repercute directamente para la mejora del currículo debido a que participar en dicho programa otorga un plus a la persona, por el hecho de haber estudiado en un lugar diferente y haber obtenido conocimientos y experiencias que resulten de provecho en un lugar de trabajo.

El presente artículo tiene por objetivo, determinar la operatividad interna del Programa de Movilidad Estudiantil en la Universidad Juárez Autónoma de Tabasco.

Antecedentes: La internacionalización universitaria no es un concepto nuevo, su origen se remonta a la edad media, en la que estudiantes españoles llegaban a Bolonia a realizar sus estudios, y es a raíz de la segunda guerra mundial, cuando cuestiones políticas y sociales constituyeron argumentos básicos para impulsarla. A finales del siglo XX, el principal objetivo para fomentarla era mejorar la calidad académica (Fernández y Ruzo, 2004).

Esta internacionalización surge como una necesidad de homologar la currícula entre las IES, a fin de fomentar el libre tránsito de personas altamente calificadas, impactando en la misión, visión y perspectivas de cada una de ellas (Gacel, 2000; Didou, 2006; Tuirán, 2009; Beneitone, 2014). Esto implica analizar la pertinencia de los programas de estudio, partiendo de la realidad que presenta cada Institución, y de su capacidad de diversificación dentro y fuera de ella, definiendo las políticas públicas, manejando agendas y priorizando actividades.

Fernández y Ruzo (2004) señalan que Instituciones de Educación aumentan sus apuestas en las estrategias de internacionalización y a las consecuencias financieras y económicas que estas conllevan. Significa que el programa fue creado con la intención de que los estudiantes, profesores y autoridades sean los actores importantes en éste; en el que se busca competir en igualdad de condiciones con todas las Instituciones involucradas en la movilidad. Dentro de las políticas educativas la movilidad estudiantil se ha convertido en una estrategia institucional idónea para elevar la calidad de la educación en colaboración internacional (Gacel, 2000).

Es importante señalar que la internacionalización de la educación superior supone una intensificación de relaciones interinstitucionales a través del mundo y la redefinición de las reglas de operación, que actualmente están dominadas por las agencias internacionales de evaluación y de acreditación (Ibarra-Colado, 2013). 
Conduciendo esto a identificar alianzas entre quienes instrumentan las políticas, definiendo de esta manera las agendas públicas nacionales, regionales, estatales y/o institucionales (Didou, 2006). Tuirán (2009), considera que "Para tener éxito en esta tarea, es imprescindible consolidar el marco institucional y los programas de formación de capital humano".

\section{Desarrollo}

El mundo entero está viviendo una vorágine de acontecimientos difícil de digerir, en la que juega un papel importante la rapidez con la que se generan los conocimientos, el avance tecnológico y científico así como las facilidades para comunicarse; exigiendo nuevos saberes, repercutiendo directamente sobre el crecimiento económico y el desarrollo nacional.

Ante estos complejos escenarios las IES requieren de modelos flexibles orientados a la solución de problemas y al trabajo en equipo que permitan la entrada y salida de sus estudiantes en diferentes momentos de su formación, teniendo la capacidad de responder a las necesidades del entorno social, cultural, económico y laboral (Ibarra-Mendívil,, 2003), por lo que en la actualidad también tienen la responsabilidad de enriquecer la comprensión de los problemas mundiales que impiden el crecimiento y desarrollo de los países.

De esta manera, se plantea la necesidad de superar las limitaciones de los países y sus instituciones, a favor de procesos de internalización. La cuestión de cómo y dónde se da la enseñanza y el aprendizaje juega un papel importante; cuando el conocimiento, la ciencia y la tecnología se priorizan en un país, se mejora notablemente su desarrollo, impactando positivamente en avances benéficos para la sociedad en general y para la economía y la educación en particular.

Al respecto, González (2011) considera que las tecnologías de la información y de la comunicación han influido en el fomento de flujos internacionales, en los que los estudiantes viajan a otro país y en algunos casos se obligan a recibir en casa a estudiantes provenientes de ese mismo país que visitaron. Además, permiten el intercambio de ideas y el almacenamiento de experiencia generando de esta manera una mayor interacción entre los estudiantes involucrados.

\section{Movilidad Académica}

La movilidad representa una alternativa para elevar la calidad académica y la competitividad internacional de alumnos, docentes e investigadores de las IES nacionales, al promover y fortalecer el entendimiento intercultural que permite la solidaridad entre los pueblos y la construcción de un clima de acercamiento mutuo (Martínez, 2004). Hoy en día se ha convertido en un factor social detonante en muchas universidades, lo que constituye un elemento importante para el desarrollo y aprendizaje integral en la formación profesional.

Los jóvenes pueden especializarse en un tema, debido a que aprenden de lugares con más información y experiencia en su área de interés. Al respecto Fresán (2009), señala que "La movilidad es una estrategia formativa cuyo objetivo rebasa el ámbito estrictamente académico y se refiere principalmente a la formación integral". La finalidad de los programas de movilidad es ofrecer a los participantes la opción de continuar sus estudios en una institución de otro país, y que éstos les sean reconocidos en la institución a la que pertenecen. (Belvis, Pineda y Moreno, 2007). Algo que es muy importante saber es ¿cuáles son los principales países del mundo a los que van los mexicanos y de donde vienen los estudiantes internacionales?

En México hay un instrumento estadístico que mide la movilidad estudiantil internacional llamado Patlani, según su base de información (Patlani, 2017), los países preferentes de destino son España, Estados Unidos, Francia, Canadá y Alemania, por otra parte, los estudiantes internacionales provienen de la Unión Americana, Francia, Colombia, España y Alemania. Cuando se comparan otras encuestas y datos, es claro que las tendencias indican que los principales países de destino son Estados Unidos, España, Francia, Canadá, Alemania y Gran Bretaña (que notoriamente destaca en destino para becarios del Conacyt (Maldonado, Cortés e Ibarra, 2016; Platani, 2017).

En otras Instituciones públicas de Educación Superior, ubicadas en la capital del país, el programa de movilidad durante el año 2016 (según datos publicados en las páginas oficiales), se atendieron 1026 estudiantes; para el periodo enero-junio 2018 han sido seleccionados 480 (Instituto Politécnico Nacional, 2017); en tanto que otra, durante ese mismo periodo otorgó (según datos publicados en su página oficial) 177 becas (Universidad Nacional Autónoma de México, 2017).

\section{Programa Institucional de Movilidad Estudiantil Internacional}

A fin de lograr exitosos programas tanto de enseñanza como de aprendizaje, esta Institución se 
apoya en un modelo educativo basado en las teorías constructivistas y humanistas centradas en la adquisición de conocimientos, es decir, el estudiante va construyendo su propio aprendizaje a partir de su propia experiencia y conocimiento enfatizando la participación y significativa (UJAT, 2015).

$\mathrm{Si}$ partimos que las Instituciones de Educación Superior de todo el mundo tienen una responsabilidad social compartida de contribuir a reducir la brecha en materia de desarrollo, mediante el aumento de conocimientos a través de las fronteras y de encontrar soluciones comunes para fomentar la circulación de competencias, este programa institucional es un claro ejemplo de los esfuerzos que esta institución realiza. (UJAT, 2012).

En este contexto, según lo planteado en el Plan Estratégico de Desarrollo 2006-2016 (2006) de esta Institución, con las tendencias de la organización, el promover una mentalidad de intercambio es determinante para tener acceso a los conocimientos más avanzados y remotos, es por eso que en esta Institución, promover la movilidad de los estudiantes es un elemento importante mediante el cual se favorecerá la actualización del campo laboral.

Este programa consiste en el intercambio de estudiantes con otras Instituciones de Educación Superior Nacionales e Internacionales, para cursar un ciclo escolar, con revalidación de créditos al finalizar su periodo escolar, debiendo cursar el último ciclo escolar de su Licenciatura en esta Universidad, que también apoya económicamente a los participantes seleccionados. De igual manera permite la recepción de estudiantes de otras Instituciones de Educación Superior que están interesados en cursar un ciclo escolar en dicha institución.

Poder participar en este programa además de ser una oportunidad, constituye una de las bases para el intercambio de experiencias y de ideas entre estudiantes de diferentes partes del mundo, que se concentran en el lugar elegido por ellos. Abonando en este sentido Haug (2010), considera que la internacionalización de los estudios ya no es optativa. Se ha convertido en una dimensión esencial del desarrollo de universidades en el ámbito competitivo en el que funciona", esto quiere decir que los estudiantes y los profesores de todos los niveles deben estar actualizados en lo que ocurre en las demás Instituciones de Educación.

Es satisfactorio saber que el $68 \%$ de sus postulantes en este programa, realizaron en el periodo agostodiciembre 2015, estancias en países como Corea del
Sur, Italia, Canadá, Brasil, España, Chile, Colombia y Costa Rica, además de 18 de las más prestigiadas universidades mexicanas. Para esta Universidad, (la pionera en la educación superior en el estado), esta cantidad de postulantes es pequeña tomando en cuenta la matrícula escolar total de 30,926 alumnos inscritos en la Institución.

Los programas de movilidad estudiantil favorecen las bases de jóvenes investigadores y talentos mexicanos a nivel nacional o internacional, por tal motivo, es importante conocer los principales factores que influyen en la toma de decisión de los estudiantes al realizar una movilidad, y sobre todo, cómo favorece el conocimiento de ellos. Además, al tener flexibilidad curricular, el estudiante tiene la ventaja de avanzar en unidades de enseñanza e ir aprendiendo a su ritmo dentro de su plan de estudios, sin necesidad de recursar las asignaturas (Fresán, 2009).

Y es que la movilidad académica internacional “...es un factor esencial para lograr el objetivo... que no es otro que eliminar las fronteras educativas entre los países... para conseguir una mejora en la calidad formativa, académica e investigadora..." (Castro y Buela-Casal, 2008, p.62). Algunos factores importantes para la elección del país destino es el apoyo que brinde la Institución Educativa de origen, contar con alguna beca que apoye el financiamiento para la estancia y los gastos que se presenten, también influyen los intereses personales y las aspiraciones profesionales.

Gacel (2007) considera que si bien se da por sentado que la Educación Superior es internacional por esencia, la movilidad de los estudiantes ha sido hasta ahora marginal, ya que aún existen obstáculos que les impiden aprovechar al máximo esta oportunidad de desarrollo, mediante el aumento de conocimientos a través de las fronteras y de encontrar soluciones comunes para fomentar la circulación de competencias, este programa institucional es un claro ejemplo de los esfuerzos que esta institución realiza. (UJAT, 2012).

\section{Material y métodos}

La presente investigación se realizó en la Universidad Juárez Autónoma de Tabasco, Institución pública que imparte estudios de licenciatura, maestría y doctorado en sus divisiones académicas, y es en la División Académica de Ciencias Económico Administrativas, donde se llevó a cabo este trabajo. 
El estudio empírico se basó en una muestra intencional de 103 cuestionarios aplicados a estudiantes cuyo avance curricular oscila entre el $50 \%$ y el $96 \%$, periodo durante el cual el universitario tabasqueño puede aplicar para participar en el programa, de igual manera se entrevistará al personal responsable de este programa, a nivel institucional y también a nivel divisional.

\section{Resultados}

Al cuestionamiento sobre si conocen o no, el programa multicitado, el 100\%, respondió afirmativamente. A ese mismo porcentaje, le gustaría participar en él. Estos jóvenes están dispuestos a demandar un espacio en el programa, aunque no se sabe en qué proporciones, este aumento modificará los índices de participación. (Altbach y
Knight, 2006; López, 2008). Cuando se quiso saber el lugar de la preferencia, las respuestas variaron de país y continente de elección. Decantándose el $6 \%$ por Asía, (Japón 2\%, China, 4\%) 31\% América, de este el $13 \%$ elegiría un país de habla no hispana. El $18 \%$, viajaría a Chile, Colombia, Cuba y Argentina. El resto, Europa.

Afortunadamente, la Institución Académica a la que pertenecen estos jóvenes tiene convenio con universidades en los países a los que ellos quieren viajar y es que "Los programas internacionales ponen al alcance de los estudiantes oportunidades para estudiar en el extranjero y entrar en contacto con otras culturas," (Altbach y Knight, 2006, p. 14). Los motivos por los que ellos deciden participar en este programa, que son variados y no exclusivamente académicos, se muestran en la tabla 1.

\begin{tabular}{|c|c|c|c|c|c|}
\hline & & Frecuencia & Porcentaje & $\begin{array}{l}\text { Porcentaje } \\
\text { válido }\end{array}$ & $\begin{array}{l}\text { Porcentaje } \\
\text { acumulado }\end{array}$ \\
\hline \multirow[t]{16}{*}{ Válido } & Zona turística & 7 & 6.8 & 6.8 & 6.8 \\
\hline & Oportunidad de trabajo & 3 & 2.9 & 2.9 & 9.7 \\
\hline & Educación de calidad & 6 & 5.8 & 5.8 & 15.5 \\
\hline & Me gusta & 16 & 15.5 & 15.5 & 31.1 \\
\hline & País desarrollado & 18 & 17.5 & 17.5 & 48.5 \\
\hline & Cultura & 24 & 23.3 & 23.3 & 71.8 \\
\hline & Mayor conocimiento & 6 & 5.8 & 5.8 & 77.7 \\
\hline & Hablan español & 5 & 4.9 & 4.9 & 82.5 \\
\hline & Crecimiento profesional & 1 & 1.0 & 1.0 & 83.5 \\
\hline & Experiencia personal & 2 & 1.9 & 1.9 & 85.4 \\
\hline & Dominar el idioma & 3 & 2.9 & 2.9 & 88.3 \\
\hline & Es interesante & 9 & 8.7 & 8.7 & 97.1 \\
\hline & Clima ambiental & 1 & 1.0 & 1.0 & 98.1 \\
\hline & Historia del país & 1 & 1.0 & 1.0 & 99.0 \\
\hline & Viajar con amigos & 1 & 1.0 & 1.0 & 100.0 \\
\hline & Total & 103 & 100.0 & 100.0 & \\
\hline
\end{tabular}

Tabla 1. Motivo de la elección

El 7\% va en plan turístico, por supuesto que debe acreditar las materias que haya matriculado a fin de justificar su estancia allá. Y es que "Una de las particularidades de la internacionalización...puede estar dada por el incremento, variedad y sofisticación de las actividades..." (Beneitone, 2014, p.33). El 3\% señala que sería una oportunidad para encontrar un mejor trabajo, otro $3 \%$ les da importancia a cuestiones sociogeográficas.

Cuando se les cuestionó sobre los beneficios que les traería el estudiar en otro país, las respuestas se muestran en la tabla 2, el total de ellos señala que estos beneficios redundarían en desarrollo académico y personal, no son meramente económicas (Altbach y Knight, 2006, p. 18; Bustos, 2016, p.143).Se observa en la tabla 2. 


\begin{tabular}{|c|c|c|c|c|c|}
\hline \multicolumn{6}{|c|}{ Beneficios } \\
\hline & & Frecuencia & Porcentaje & $\begin{array}{l}\text { Porcentaje } \\
\text { válido }\end{array}$ & $\begin{array}{l}\text { Porcentaje } \\
\text { acumulado }\end{array}$ \\
\hline \multirow{12}{*}{ Válido } & Nuevos pensamientos & 7 & 6.8 & 6.8 & 6.8 \\
\hline & Diferentes metodologías & 8 & 7.8 & 7.8 & 14.6 \\
\hline & Mayor conocimiento & 29 & 28.2 & 28.2 & 42.7 \\
\hline & Cultura & 37 & 35.9 & 35.9 & 78.6 \\
\hline & Perfeccionar el idioma & 9 & 8.7 & 8.7 & 87.4 \\
\hline & Desarrollo personal & 3 & 2.9 & 2.9 & 90.3 \\
\hline & Conocimiento político & 1 & 1.0 & 1.0 & 91.3 \\
\hline & Calidad en el Currículum Vitae & 1 & 1.0 & 1.0 & 92.2 \\
\hline & Mayor oportunidad laboral & 4 & 3.9 & 3.9 & 96.1 \\
\hline & Obtener becas estudiantiles & 2 & 1.9 & 1.9 & 98.1 \\
\hline & Mayor aprendizaje & 2 & 1.9 & 1.9 & 100.0 \\
\hline & Total & 103 & 100.0 & 100.0 & \\
\hline
\end{tabular}

Tabla 2. Beneficios de estudiar en otro país

$\mathrm{Al}$ cuestionamiento sobre si tiene algún impedimento para participar en el Programa, el 60\% considera que sí. Entre los motivos que presentan son: la familia (29\%), cuestiones económicas (55\%), el resto, señala como impedimentos terminar la licenciatura, no dominar el idioma, y el promedio académico.

\section{Discusión}

Los resultados exponen una homogeneidad en las intenciones, de parte de los estudiantes, para ser participantes de los programas de becas para realizar el intercambio y/o movilidad estudiantil. Esto habla acerca de dinamismo universitario y de la buena recepción que goza el programa de intercambio estudiantil.

$\mathrm{Al}$ adentrarse en la información obtenida, se registra una serie de motivos para aplicar al programa de movilidad; si bien se trata de un variado y heterogéneo grupo de motivos, esto no resta validez al propósito del programa, pues además de que dentro de los lineamientos del programa se establecen las regulaciones necesarias para poder brindarle la oportunidad a los estudiantes más competentes y que estos tengan el mejor aprovechamiento académico, estos motivos también persiguen la integración e intercambio de experiencias, de cultura $\mathrm{y}$, por supuesto, de conocimiento, se concuerda con otros estudios realizados por (Belvis, Pineda, y Moreno 2007).

Se observa que los estudiantes son capaces de identificar una serie de beneficios que el programa de movilidad puede integrar en su carrera profesional y vida personal. Aquí se presenta una variedad de beneficios, que en percepción de los estudiantes, son resultado al ser beneficiario del programa de movilidad. Igualmente se percibe que los motivos van desde lo personal hasta lo académico que, en su totalidad, hacen aporte positivo en la formación profesional de los estudiantes, que al final, es de los aspectos principales que se persigue con el establecimiento de estos programas, se coincide con (García 2013).

Como último, se identifican obstáculos que el estudiante puede presentar y que pueden impedir su participación activa en los programas de movilidad. Estos obstáculos, si bien algunos escapan del ámbito de competencia académico, como lo son aquellos que se presentan en el ámbito familiar; se observan aquellos en los que es posible realizar una intervención para su resolución, como lo son los de aspectos económicos, que representan más de la mitad de la muestra de los resultados obtenidos.

Así también los hay de carácter académico, pues aunque algunos van desde la insuficiencia del promedio, que se usa como filtro para beneficio de los estudiantes con mejor aprovechamiento, también se registra la incompatibilidad del lenguaje y el deseo de concluir con prontitud sus estudios universitarios. Estos obstáculos, casi en su totalidad, pueden resolverse con promoción e incitación oportuna, pues aunque para participar en este programa se necesita tener un avance curricular propio de estudiantes de cursos avanzados, estos aspectos deben tomarse en cuenta a temprana o de preferencia a inicios de su carrera profesional, se concuerda con otras investigaciones (Bustos, 2016). 


\section{Conclusiones}

El programa de Movilidad estudiantil Internacional es una estrategia para la Universidad Juárez Autónoma de Tabasco, que busca impulsar la formación académica, abriendo paso a la competitividad que exige el campo laboral. Sin lugar a duda, el impacto que tiene este programa no puede perderse de vista, ya que la operatividad interna no solo se enfoca en fortalecer los procesos de enseñanza-aprendizaje, sino en elevar los estándares de calidad que requieren las IES.

En lo que respecta a la UJAT como Institución emisora, los resultados obtenidos muestran que la movilidad ha progresado a lo largo del presente siglo, no obstante, se deberían incrementar los montos otorgados debido a que no todos los participantes cuentan con recursos suficientes para sustentarse en otro país con una economía pujante, del mismo modo, deberían otorgar el apoyo económico en tiempo y forma para que el alumno no tenga algún imprevisto que pueda influir en su participación, aunque la universidad no se responsabiliza del gasto del seguro médico ni del hospedaje, debería cubrir gran parte de ese pago y sugerirle al participante opciones de vivienda durante su estancia académica, asimismo se debe afinar los convenios con las IES extranjeras que permitan la internalización de la universidad.

Como se desprende del análisis del cuestionario, es un factor crucial que el $100 \%$ de los entrevistados conozcan y quieran participar en el programa, sin embargo, el 60\% tiene impedimentos que van desde motivos personales hasta económicos, otro obstáculo es no dominar un idioma diferente al del habla hispana.

La movilidad estudiantil internacional es una herramienta que necesita de la participación de todos los agentes educativos, el reto es grande, ya que es preciso concientizar a la comunidad estudiantil de mantener un buen promedio, de reforzarles la importancia de obtener una certificación de un idioma extranjero y de difundir los beneficios que tiene el programa. Esta investigación abre paso a otras investigaciones, el darle seguimiento al alumno durante su estancia, el hacer análisis sobre el progreso del programa, el realizar estudios comparativos con los participantes y no participantes.

\section{Bibliografía}

Altbach, P. y Knight, J. (2006). Visión panorámica de la internalización en la educación superior: motivaciones y realidades. Perfiles Educativos, 28 (112).

Belvis, E., Pineda, P. y Moreno, M. V. (2007). La participación de los estudiantes universitarios en programas de movilidad: factores y motivos que la determinan. Revista Iberoamericana de Educación, 42 (5), 1-14.

Beneitone, P. (2014). De la Cooperación Internacional Universitaria a la Internacionalización de la Educación Superior: ¿cambio de paradigma o maquillaje conceptual?. En Tangelson, Guillermo. (Compilador). Desde el Sur: miradas sobre la internacionalización, 29- 38. Argentina: Universidad Nacional de Lanús.

Bustos, M. L. (2016). La movilidad Internacional y el desarrollo de competencias para el empleo: una revisión de la literatura. 141- 150. En Desafíos en el campo de las investigaciones de trayectoria estudiantil, egresados y mercados laborales.

Castro, A. y Buela-Casal, G. (2008). La movilidad de profesores y estudiantes en programas de postgrado: ranking de las universidades españolas. Revista de Investigación en Educación, 5 (2008), 61-74.

Didou, S. (2006). Internacionalización de la educación superior y provisión transnacional de servicios educativos en América Latina: del voluntarismo a las elecciones estratégicas. Venezuela: Unesdoc

Fernández, S. y Ruzo, E. (2004). Los procesos de internacionalización y regionalización de la educación superior: un análisis de los países OCDE. Revista de Educación, 335, 385-413.

Fresán, M. (2009). Impacto del programa de movilidad académica en la formación integral de los alumnos. Revista de la Educación Superior, 38 (3), 141-160.

Gacel, J. (2000). La dimensión internacional de las universidades mexicanas. Educación Superior y Sociedad, 115 (1), 121-142.

Gacel, J. (2007). Calidad y educación sin fronteras. Apertura, 7 (6), 76-91.

García, J. J. (2013). Movilidad estudiantil internacional y cooperación educativa en el nivel superior de educación. Revista Iberoamericana De Educación, 61, 59-76.

González, A. M. (2011). Atrayendo talento: Estrategias de movilidad de los profesionales 
altamente cualificados en España. Sociología y Tecnociencia. Revista digital de sociología del sistema científico, 1 (2), 72-87.

Haug, G. (2010). La internacionalización de la educación superior: más allá de la movilidad europea. La cuestión universitaria, 6, 20-29.

Ibarra-Colado, E. (2013). Políticas gubernamentales, universidades públicas y racionalidad liberal. Relevancia del gobierno y la gestión institucional. Diálogos sobre educación, 4 (6), $1-13$.

Ibarra-Mendívil， J. L. (2003). La universidad necesaria. Revista electrónica de investigación educativa, 5 (1), 186-193.

Instituto Politécnico Nacional (2017). Alumnos de nivel superior asignados en movilidad internacional periodo enero-junio 2018. Fecha de consulta: 16/octubre/2017. http://bit.ly/2LPc72e

López, F. (2008). Tendencias de la educación superior en el mundo y en América Latina y el Caribe. Revista da Avaliação da Educação Superior (Campinas), 13 (2), 267-291.

Maldonado, A., Cortés, C. e Ibarra, B. (2016). Platani. Encuesta mexicana de movilidad internacional estudiantil, 2012/13 y 2'13/14. México: ANUIES, 113-119.

Martínez, J. B. (2004). Movilidad / movilización de profesorado y estudiantes para la formación. Revista Interuniversitaria de Formación del Profesorado, 18 (3), 233-250.

Patlani (2017). PATLANI: Encuesta Nacional de Movilidad Estudiantil Internacional 2012 2013 / 2013 - 2014 ANUIES. [http://patlani.anuies.mx/login.php

Tuirán, R. (2009). Fuga de cerebros: algunos comentarios a partir del caso mexicano. En Didou, S. y Etienne, G. (Editores). Fuga de cerebros, movilidad académica, redes científicas. Perspectivas latinoamericanas. México: IESALC-CINVESTAV-IRD

Torres, H., Medina, A. y Alvarado, G. (2019). La movilidad estudiantil: agente de formación personal, profesional y fuente curricular para el mejoramiento del perfil de egreso. Recuperado de

UNESCO (2015). UNESCO Science Report: towards 2030 - Executive Summary. (Informe de la UNESCO sobre la ciencia: hacia el 2030 - Resumen ejecutivo). Recuperado de
Universidad Juárez Autónoma de Tabasco (2006). Plan Estratégico de desarrollo 20062016.México: Colección Justo Sierra.

Universidad Juárez Autónoma de Tabasco (2012). Plan de Desarrollo Institucional 2012-2016. México: Colección Justo Sierra.

Universidad Juárez Autónoma de Tabasco (2015). Plan de Desarrollo a largo plazo 2028. México: Colección Justo Sierra.

Universidad Nacional Autónoma de México (2017). Movilidad Internacional Estudiantil SEP-UNAMFUNAM 2017 2da fase. Fecha de consulta: 15/octubre/2017. http://bit.ly/2Os1xzA 